their disease. The patients were randomized to either a control group, or an experimental group; these patients were prescribed a supplemented vegan diet with daily exercise, stress-management techniques, and a weekly support meeting.

After 1 year, six patients from the control group, but none from the experimental group, had undergone conventional treatment. PSA had decreased by $4 \%$ on average in the experimental group, compared with a $6 \%$ increase in the control group $(P=0.016)$. LNCaP cell growth was inhibited by $70 \%$ and $9 \%$ by serum from experimental-group patients and controlgroup patients, respectively $(P=0.001)$, suggesting that positive lifestyle changes might indeed slow the progression of prostate cancer. Furthermore, patients in the experimental group demonstrated significant weight loss and an improved lipid profile that reduced their risk of cardiovascular disease.

Owing to the short follow-up time, dramatic changes in clinical progression were unlikely. Further down the line, the authors plan to report on rates of recurrence, metastasis, and death. Tamsin Osborne

Original article Ornish D et al. (2005) Intensive lifestyle changes may affect the progression of prostate cancer. J Urol 174: $1065-1070$

\section{Does cycling lead to erectile dysfunction?}

When investigating patients with erectile dysfunction (ED), Huang and colleagues observed a high incidence of blunt perineal trauma, often associated with cycling. Concern regarding this association has grown over the past two decades, prompting the team to summarize the data published on the subject and to suggest measures that cyclists could use to minimize the risk of ED. The authors looked at case reports, observational series, casecontrol studies, mechanistic investigations, and population-based epidemiologic studies.

The literature revealed that the rider's interaction with the saddle seems to be related to the development of ED: straddling the saddle leads to perineal compressive pressures and occludes penile blood flow. Acute traumafalling on the crossbar, for example-can also contribute to the development of ED.
Based on their findings, the authors recommend that cyclists opt for wider saddles without protruding front extensions, which have been shown to reduce penile blood flow, and tilt the front of the saddle downwards to reduce perineal pressure. Gel saddles have been shown to promote better penile oxygenation than foam saddles, as has adopting a more upright or reclining position. Better still, cyclists could replace traditional bicycles with recumbent bicycles, which are not associated with ED.

The authors note that perineal compression time can be reduced by cycling less often or standing up on the pedals, but discourage giving up cycling altogether owing to its cardiovascular benefits, which actually protect against $\mathrm{ED}$, as well as the benefits to the environment.

Tamsin Osborne

Original article Huang V et al. (2005) Bicycle riding and erectile dysfunction: an increase in interest (and concern). J Sex Med 2: 596-604

\section{Antiandrogen and vaccine therapy for hormone-refractory prostate cancer}

Researchers at the National Cancer Institute, MD, USA, have investigated the combination of antiandrogen and vaccine therapy for the treatment of nonmetastatic hormone-refractory prostate cancer.

Patients who had received prior hormone therapy and had increasing prostate specific-antigen (PSA) levels, but no evidence of metastases, were randomized to receive second-line antiandrogen therapy with nilutamide, or a vaccination regimen comprising recombinant vaccinia viruses with $P S A$ and $B 7.1$ co-stimulatory genes, and the avipox-PSA vaccine as a booster. There was a significant decrease in the estimated PSA velocity after 6 months in patients receiving nilutamide $(P=0.009)$, but the decrease was nonsignificant in the vaccine arm. The time to treatment failure in each arm did not differ significantly. Patients whose PSA levels continued to rise, but who had no metastatic progression, were then given the option to receive therapy from the other treatment arm combined with their existing regimen. This strategy was adopted in $12 / 21$ patients in the vaccine arm and 8/21 patients in the 